Article

\title{
A Route to Dicyanomethylene Pyridines and Substituted Benzonitriles Utilizing Malononitrile Dimer as a Precursor
}

\author{
Noha M. Helmy ${ }^{1, *}$, Fatma E. M. El-Baih ${ }^{1}$, Monirah A. Al-Alshaikh ${ }^{1}$ and Moustafa S. Moustafa ${ }^{2}$ \\ 1 Women Students-Medical Studies \& Sciences Sections, Chemistry Department, College of Science, \\ King Saud University, Riyadh, KSA, P.O. Box 22452, Riyadh 11495, Saudi Arabia \\ 2 Department of Chemistry, Faculty of Science, University of Kuwait, Safat 13060, Kuwait \\ * Author to whom correspondence should be addressed; E-Mail: Elnagdinoha@yahoo.com; \\ Tel.: +966-500432102; Fax: +966-1 477-2245.
}

Received: 12 October 2010; in revised form: 20 December 2010 / Accepted: 22 December 2010 / Published: 4 January 2011

\begin{abstract}
The conditions of the reaction of malononitrile dimer with enaminones and arylidenemalononitrile could be adapted to yield either pyridines or benzene derivatives. A new synthesis of pyrido[1,2-a]pyrimidines from the reaction of malononitrile dimer $\mathbf{1}$ and 2-phenyl-3-piperidin-1-yl-acrylonitrile (11) is described. Compound 1 condensed with DMFDMA to yield an enaminonitrile that reacted with hydrazine hydrate to yield $N^{\top}, 4,6$ triamino-2H-pyrazolo[3,4-b]pyridine-5-carboxamidine (17).
\end{abstract}

Keywords: malononitrile dimer; arlymethylenemalononitrile; benzylidenemalononitrile; pyrazolopyridine

\section{Introduction}

Polyfunctionally substituted nitriles are versatile reagents that have been extensively utilized in the past as precursors to polyfunctionally substituted heteroaromatics [1-4]. Interest in further developing the synthetic potential of these compounds has been revived [5-7]. 2-Aminoprop-1-ene-1,1,3tricarbonitrile (1) has proved to be an excellent precursor to condensed pyridines, pyridazines, and pyrazoles [8-10]. However, to our knowledge the utility of $\mathbf{1}$ as a precursor to polyfunctional aromatics has received little attention. Elnagdi et al. have noted the formation of $\mathbf{3}$ as a side product from the reaction of 2 with 1, while compound 4 was obtained as the main product [11] (Scheme 1). In 
connection to results reported earlier [11] we were able to react $\mathbf{1}$ with $\mathbf{5 a , b}$ to afford either pyridines or benzene derivatives after changing the reaction conditions.

Scheme 1. Malononitrile dimer as precursor to heterocycles and substituted benzenes [11].

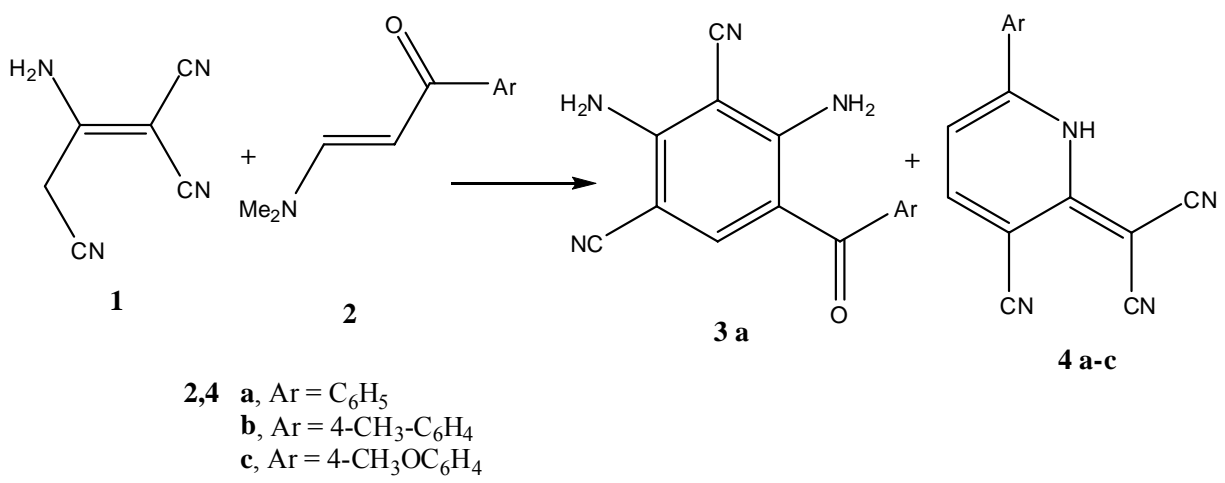

\section{Results and Discussion}

Thus reaction of $\mathbf{1}$ with 2a,b in ethanolic piperidine has afforded $\mathbf{4 a , d}$ as a sole product. On the other hand, when the reaction was conducted in acetic acid in the presence of ammonium acetate and refluxing for $4 \mathrm{hrs}$ only 3a was formed via intermediate 5 (Scheme 2) The ${ }^{1} \mathrm{H}-\mathrm{NMR}$ of $\mathbf{4 a}$, in addition to phenyl proton signals, showed two doublets at $\delta=7.16 \mathrm{ppm}$ and $\delta=7.86 \mathrm{ppm}$ with $J=8 \mathrm{~Hz}$, typical for pyridine $\mathrm{H}-5$ and $\mathrm{H}-4$, respectively. $\mathrm{A}_{2} \mathrm{O}$ exchangeable one proton signal for a $\mathrm{NH}$ group appeared at $\delta=9.42 \mathrm{ppm}$.

Scheme 2. Synthesis of pyridine 3a and substituted benzenes 4a,d.

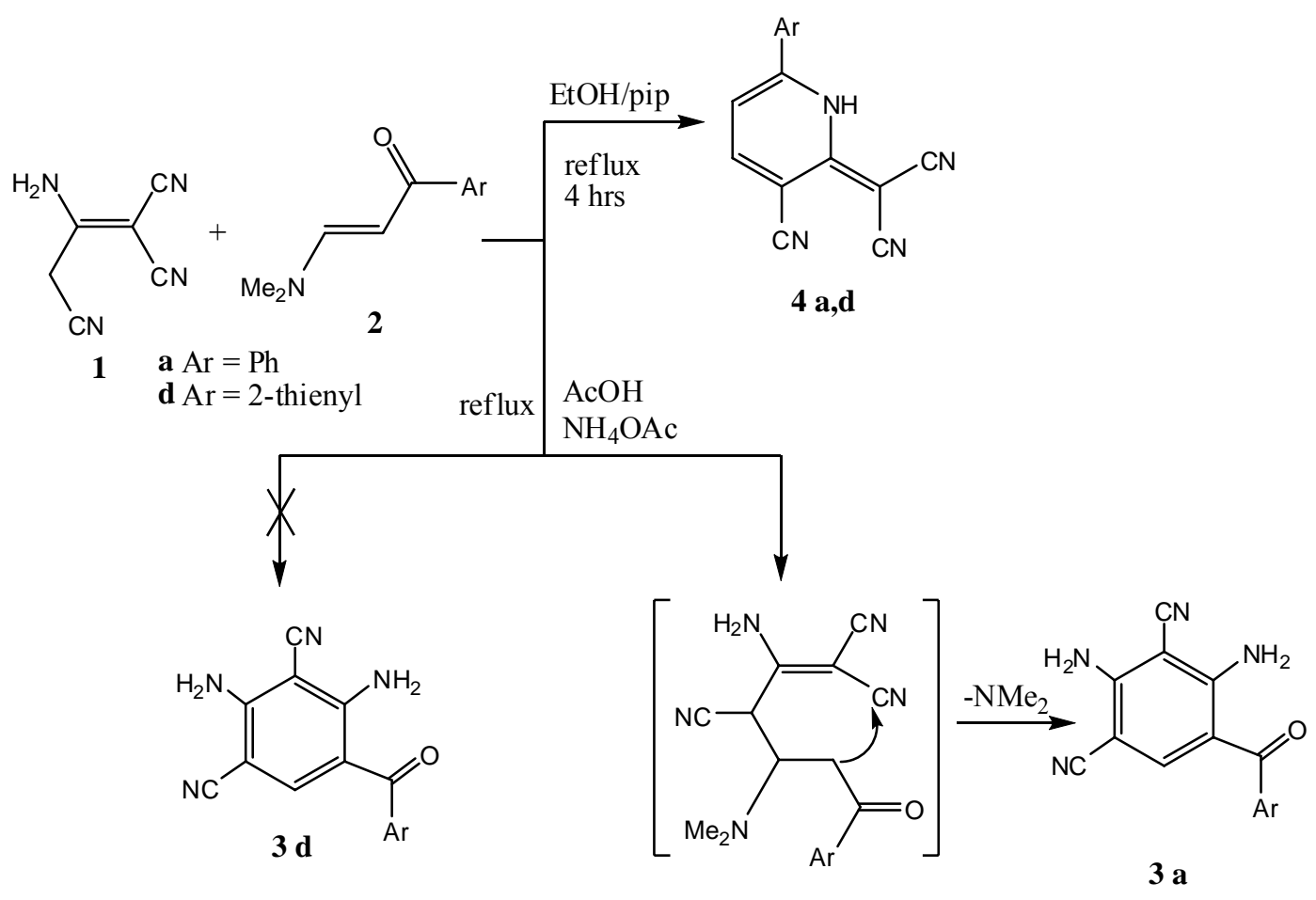


The ${ }^{1} \mathrm{H}-\mathrm{NMR}$ spectrum of 3a revealed a singlet at $\delta=8.29 \mathrm{ppm}$ for $\mathrm{H}-6$ and two $\mathrm{D}_{2} \mathrm{O}$ exchangeable amino signals at $\delta=7.08 \mathrm{ppm}$ and $\delta=7.77 \mathrm{ppm}$, in addition to the phenyl protons (see Experimental). The ${ }^{13} \mathrm{C}-\mathrm{NMR}$ clearly indicated the carbonyl carbon at $\delta=190.23 \mathrm{ppm}$ and two $\mathrm{CN}$ signals at $\delta=114.13$ and $113.85 \mathrm{ppm}$.

In an attempt to generate further examples of the synthesis of substituted benzenes 3, malononitrile dimer $\mathbf{1}$ was reacted with $\mathbf{2 d}$ in acetic acid/ammonium acetate, and a product with molecular formula $\mathrm{C}_{13} \mathrm{H}_{8} \mathrm{~N}_{4} \mathrm{OS}\left(\mathrm{M}^{+}\right.$at $\left.m / z=268\right)$ which we think to be $3 \mathbf{d}$ was isolated after reflux for $1 / 2 \mathrm{hrs}$; prolonged heating did not change the identity of the compound. The ${ }^{1} \mathrm{H}-\mathrm{NMR}$ under $\mathrm{D}_{2} \mathrm{O}$ exchange of the presumed structure 3d; showed, along with three thienyl protons, two amino group singlets at $\delta=7.1 \mathrm{ppm}$ and $\delta=7.9 \mathrm{ppm}$, and a doublet at $\delta=8.2 \mathrm{ppm}$ with $J=8 \mathrm{~Hz}$, that could not be explained or assigned to any proton in the suggested structure.

Repeating the same reaction using sodium acetate instead of ammonium acetate, and refluxing for 3 hrs, a whole new set of data were obtained. A compound with molecular formula $\mathrm{C}_{20} \mathrm{H}_{12} \mathrm{~N}_{4} \mathrm{O}_{2} \mathrm{~S}_{2}\left(\mathrm{M}^{+}\right.$ 404) was obtained. ${ }^{1} \mathrm{H}-\mathrm{NMR}$ of this compound showed two singlets at $\delta=7.47 \mathrm{ppm}$ and $\delta=8.98 \mathrm{ppm}$ each for one proton of $\mathrm{C}-5$ and $\mathrm{C}-2$ of the pyridine ring, respectively, in addition to six thienyl protons and two amino signals. The ${ }^{13} \mathrm{C}-\mathrm{NMR}$ spectrum showed the presence of 19 different carbon atoms with two carbonyl carbons at $\delta=184.7 \mathrm{ppm}$. These data can be interpreted as corresponding to structure 7 that is assumed to result from initial reaction of the active methylene moiety and the amino function in $\mathbf{1}$ with $\mathbf{2 d}$ to yield the intermediate $\mathbf{6}$ that then cylizes to $\mathbf{7}$ (Scheme 3).

Scheme 3. Synthesis of 4,7-diamino-3,6-di(thiophene-2-carbonyl)quinoline-8-carbonitrile (7).

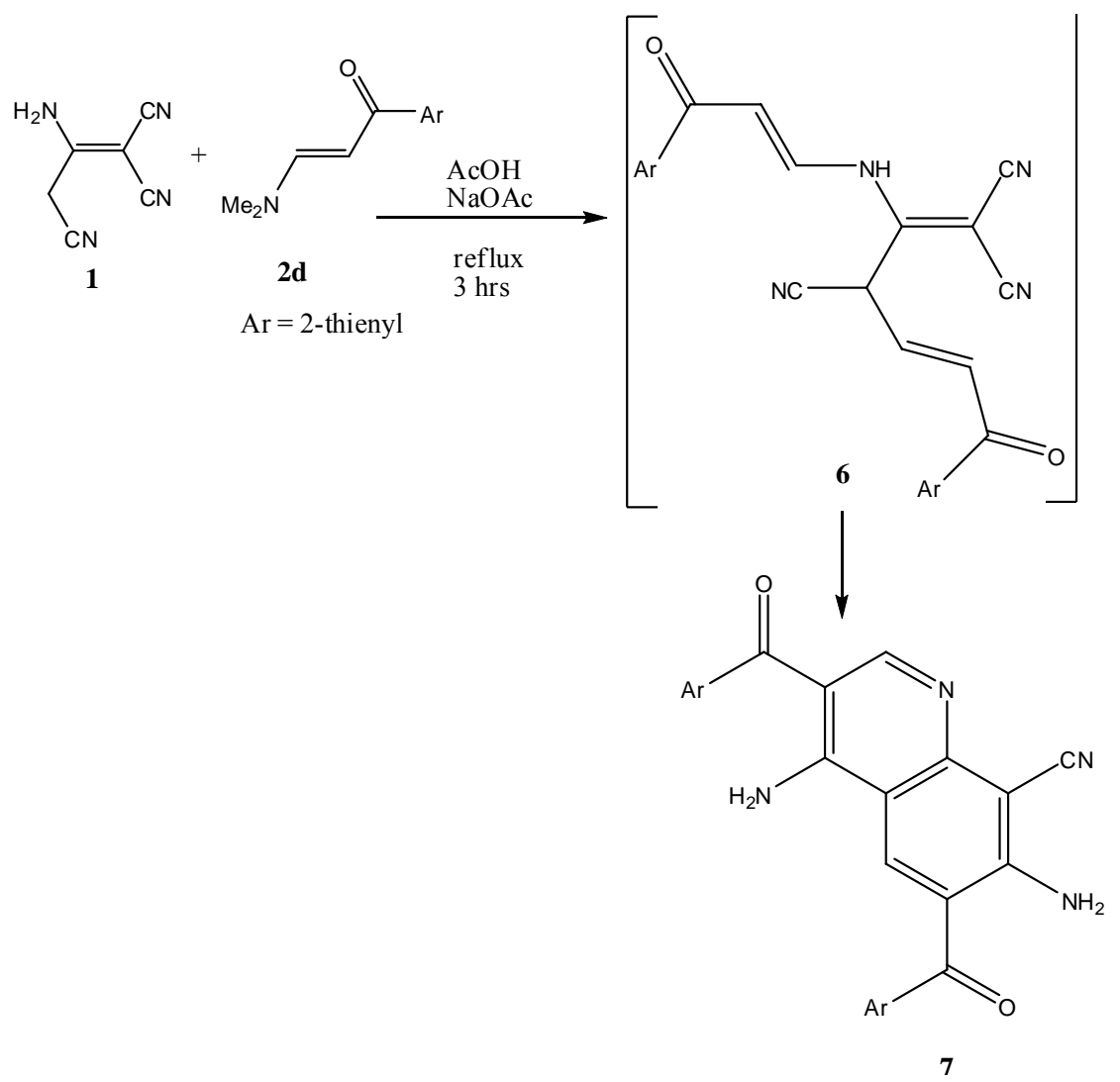


Like the recently reported formation of $\mathbf{9}$ from reaction of $\mathbf{1}$ and $\mathbf{8 a} \mathbf{b}$ in ethanolic chitosan, compound 1 reacted with $\mathbf{8 a}, \mathbf{b}$ to yield dihydropyridine 9a,b. However in refluxing acetic acid in the presence of ammonium acetate, the benzene derivative $\mathbf{1 0}$ was obtained as indicated by the spectral data (Scheme 4).

Scheme 4. Synthesis of 6-amino-2-dicyanomethylene-4-aryl-2,3-dihydropyridine-3,5dicarbonitriles 9a,b and 3,5-diaminobiphenyl-2,4,6-tricarbonitrile (10).

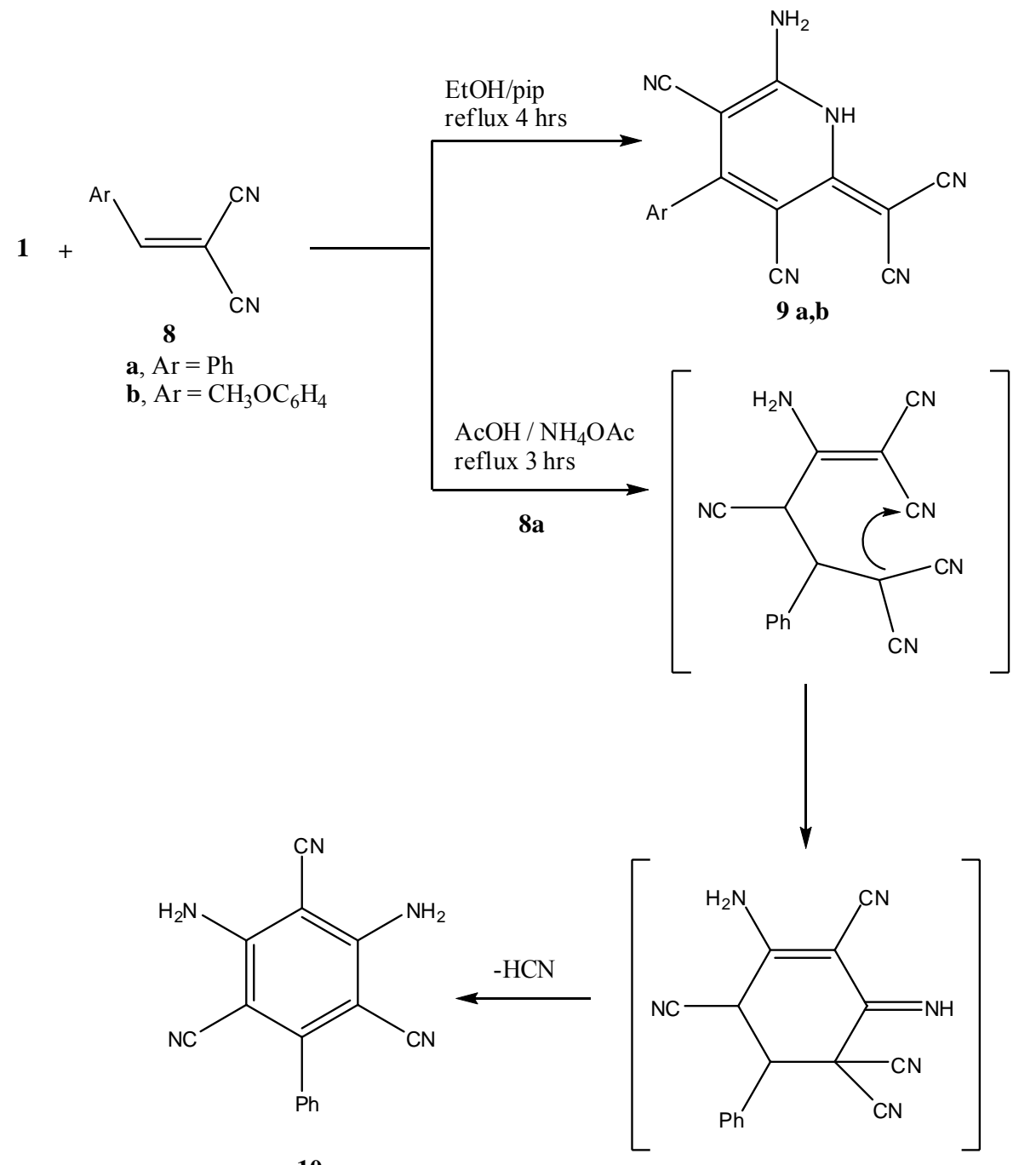

10

The reaction of 1 with 11, which was recently obtained by reacting benzyl cyanide with triethyl orthoformate and piperidine [12], afforded 14 via intermediates $\mathbf{1 2}$ and $\mathbf{1 3}$. Attempts to isolate $\mathbf{1 3}$ have failed (cf. Scheme 5). The reaction of 1 with DMFDMA afforded 16 which may exist in $E$ or $Z$ forms. Isomeric structure 15 was ruled out based on ${ }^{1} \mathrm{H}$ NMR that revealed the $\mathrm{D}_{2} \mathrm{O}$ exchangeable amino signal at $\delta=7.19 \mathrm{ppm}$. In addition, the ${ }^{13} \mathrm{C}$ NMR did not reveal any signals for the $\mathrm{sp}^{3}$ carbons other than those of the dimethylamino moiety. Reacting 16 with hydrazine hydrate afforded 4,6-diamino2H-pyrazolo[3,4-b]pyridine-5-carboxamide hydrazone 17 (cf. scheme 6). 
Scheme 5. Synthesis of 2-(4-amino-7-cyano-3,9-diphenyl-pyrido[1,2-a]pyrimidin-6ylidene)-malononitrile (14).

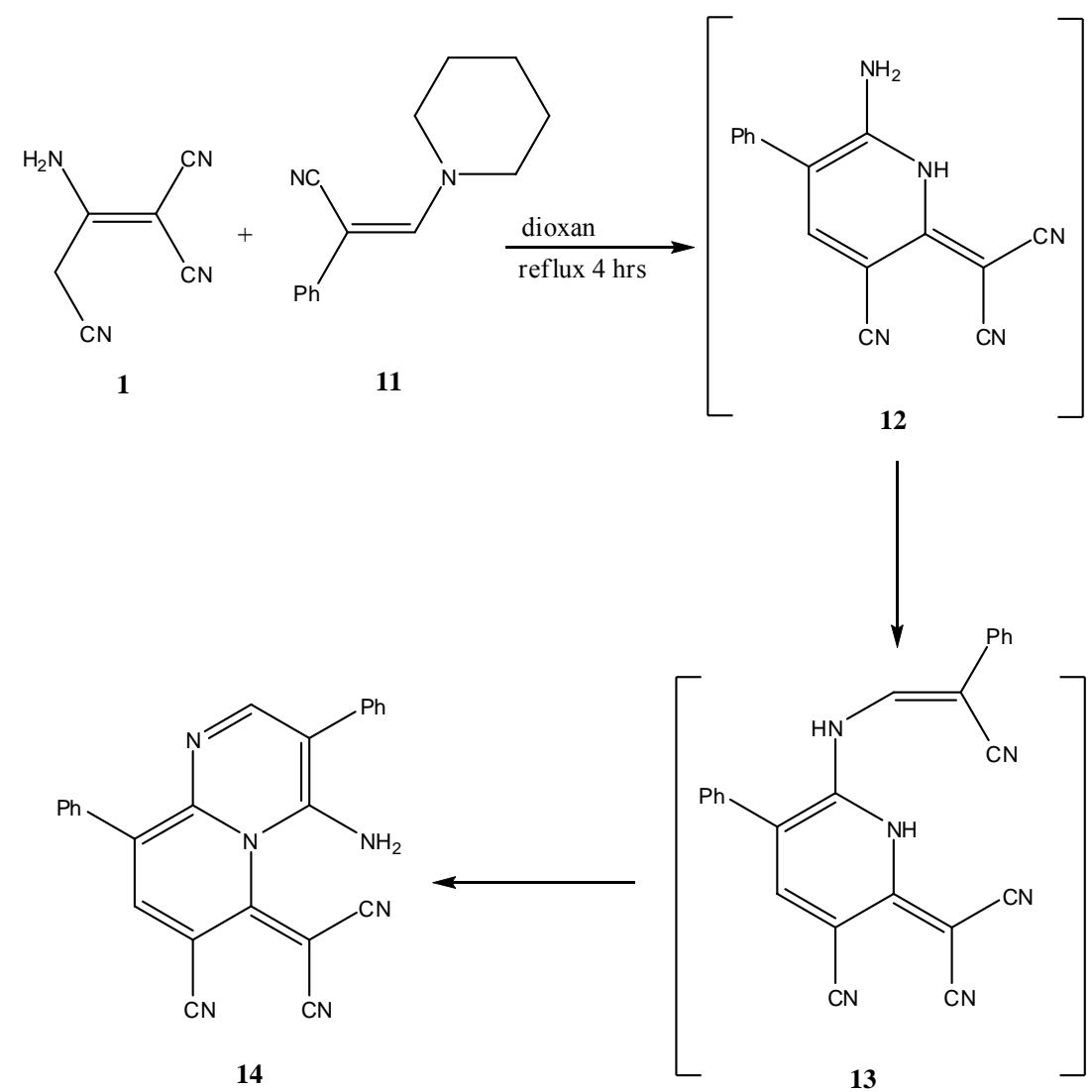

Scheme 6. Synthesis of 4,6-diamino-2H-pyrazolo[3,4-b]pyridine-5-carboxamide hydrazone (17).

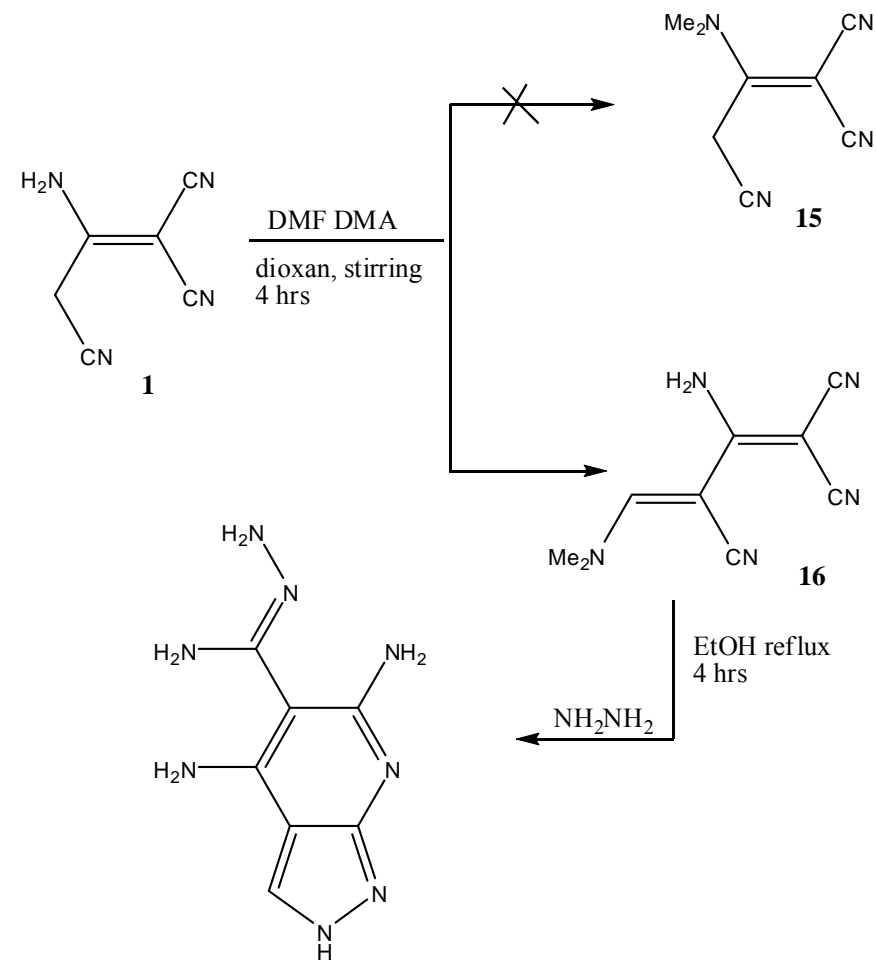




\section{Experimental}

\subsection{General}

All melting points are uncorrected and were determined on a Sanyo (Gallenkamp) instrument. Infrared spectra were recorded from $\mathrm{KBr}$ discs on a Perkin-Elmer $2000 \mathrm{FT}-\mathrm{IR}$ system. ${ }^{1} \mathrm{H}-\mathrm{NMR}$ and ${ }^{13} \mathrm{C}-\mathrm{NMR}$ spectra were determined on a Bruker DPX spectrometer operating at $400 \mathrm{MHz}$ for ${ }^{1} \mathrm{H}-\mathrm{NMR}$ and $100 \mathrm{MHz}$ for ${ }^{13} \mathrm{C}$-NMR using DMSO- $\mathrm{d}_{6}$ as solvent and TMS as internal standard; chemical shifts are reported in $\delta$ (ppm). Mass spectra were measured on VG Autospec Q MS 30 and MS 9 (AEI) spectrometers, with EI at $70 \mathrm{eV}^{\prime}$. Elemental analyses were measured by means of LEOCHNS-932 Elemental Analyzer. General purpose silica gel on polyester 20 × $20 \mathrm{~cm}$ TLC plates with UV indicator were used in TLC experiments to monitor completion of reactions, in which ethyl acetate-petroleum ether $(1: 1)$ was used as eluent.

\subsection{2,4-Diamino-5-benzoyl-isophthalonitrile (3a)}

A mixture of 2-aminoprop-1-ene-1,1,3-tricarbonitrile (1, $1.32 \mathrm{~g}, 0.01 \mathrm{~mol})$ and enaminone 2a $(0.01 \mathrm{~mol})$ in $\mathrm{AcOH}(10 \mathrm{~cm})$ and $0.2 \mathrm{gm}$ of $\mathrm{NH}_{4} \mathrm{OAc}$, was kept at reflux temperature for $4 \mathrm{hrs}$. The mixture was cooled and then poured onto ice-water. The solid, so formed, was collected by filtration and recrystallized from EtOH to give yellow crystals; Yield 83\%; m.p. $298-299{ }^{\circ} \mathrm{C}$; Anal. Calcd. for $\mathrm{C}_{15} \mathrm{H}_{10} \mathrm{~N}_{4} \mathrm{O}$ (262.27): C, 68.69; H, 3.84; N, 21.36\%. Found: C, 68.53; H, 3.92; N, 21.34\%; IR (KBr, $\left.\mathrm{cm}^{-1}\right): 3,443,3,352\left(\mathrm{NH}_{2}\right), 3,322,3,209\left(\mathrm{NH}_{2}\right), 2,210,2,206(2 \mathrm{CN}) ;{ }^{1} \mathrm{H}-\mathrm{NMR}: \delta, \mathrm{ppm}=7.08$ (br s, $2 \mathrm{H}$, $\mathrm{NH}_{2}, \mathrm{D}_{2} \mathrm{O}$ exchangeable) 7.50-7.57 (3H, m, H-3',4',5'), 7.77 ( $2 \mathrm{H}$, br. s, $\mathrm{NH}_{2}, \mathrm{D}_{2} \mathrm{O}$ exchangeable), 8.22 $\left(2 \mathrm{H}, \mathrm{dd},{ }^{3} J=8.0,{ }^{4} J=1.6, \mathrm{H}-2^{\prime}, 66^{\prime}\right), 8.29(1 \mathrm{H}, \mathrm{s}, \mathrm{H}-6) ;{ }^{13} \mathrm{C}-\mathrm{NMR}: \delta, \mathrm{ppm}=190.23,159.85,159.62$, 157.90, 154.44, 154.12, 143.65, 136.01, 130.21 (2C), 127.32 (2C), 115.89, 114.13, 113.85; MS: $\mathrm{m} / \mathrm{z}$ (\%) $262\left(\mathrm{M}^{+}, 100\right), 234$ (15), 217 (5), 192 (5), 164 (25), 131 (10).

\subsection{General procedure for the synthesis of compounds $\mathbf{4 a , d}$}

A mixture of 2-aminoprop-1-ene-1,1,3-tricarbonitrile (1, $1.32 \mathrm{~g}, 0.01 \mathrm{~mol})$ and enaminone 2a,b $(0.01 \mathrm{~mol})$ in EtOH $(10 \mathrm{~mL})$ was treated with piperidine (5 drops). The reaction mixture was refluxed for $4 \mathrm{~h}$. The mixture was cooled and then poured onto ice-water. The solid, so formed, was collected by filtration and recrystallized from EtOH to give yellow crystals.

2-(3-Cyano-6-phenyl-1H-pyridin-2-ylidene)malononitrile (4a). Yield 80\%; m.p. 257-259 ${ }^{\circ} \mathrm{C}$; Anal. Calcd. for $\mathrm{C}_{15} \mathrm{H}_{8} \mathrm{~N}_{4}$ (244.26): C, 73.76; H, 3.30; N, 22.94\%. Found: C, 73.94; H, 3.52; N, 23.04\%; IR $\left(\mathrm{KBr}, \mathrm{cm}^{-1}\right): 3,097(\mathrm{NH}), 2,210,2,182(3 \mathrm{CN}) ;{ }^{1} \mathrm{H}-\mathrm{NMR}: \delta, \mathrm{ppm}=7.16(1 \mathrm{H}, \mathrm{d}, J=8.0 \mathrm{~Hz}, \mathrm{H}-5)$, 7.48-7.51 (3H, m, H-3',4',5'), 7.86 (1H, d, J=8.0 Hz, H-4), 8.01 (2H, m, H-2',6'), 9.42 (1H, br. s, NH, $\mathrm{D}_{2} \mathrm{O}$ exchangeable); ${ }^{13} \mathrm{C}-\mathrm{NMR}: \delta, \mathrm{ppm}=180.88,160.54,159.45,155.50,136.89,132.09,128.31(2 \mathrm{C})$, 127.32 (2C), 114.72, 113.88 (2C), 91.82, 63.25; MS: $m / z(\%) 243\left(\mathrm{M}^{+}, 100\right), 217$ (25), 152 (25), 128 (15), 105 (100), 77 (10).

2-(3-Cyano-6-thiophen-2-yl-1H-pyrid in-2-ylidene)malononitrile (4d). Yield 75\%; m.p. 200-202 ${ }^{\circ} \mathrm{C}$; Anal. Calcd. for $\mathrm{C}_{13} \mathrm{H}_{6} \mathrm{~N}_{4} \mathrm{~S}$ (250.28): C, 62.93; H, 2.42; N, 22.39; S, 12.81\%. Found: C, 63.06; H, 2.54; 
N, 22.54; S, 12.98\%; IR (KBr, $\left.\mathrm{cm}^{-1}\right): 3,189(\mathrm{NH}), 2,230,2,210(3 \mathrm{CN}) ;{ }^{1} \mathrm{H}-\mathrm{NMR}: \delta, \mathrm{ppm}=7.04(1 \mathrm{H}$, $\mathrm{d}, J=8.0, \mathrm{H}-5), 7.14(1 \mathrm{H}, \mathrm{t}, J=4.0$, thienyl H-4'), 7.66-7.68 (2H, m, H-4, thienyl H-3'), $7.76(1 \mathrm{H}, \mathrm{d}$, $J=4.0$, thienyl $\left.\mathrm{H}^{-5}\right), 8.06\left(1 \mathrm{H}\right.$, br. s, $\mathrm{NH}, \mathrm{D}_{2} \mathrm{O}$ exchangeable); MS: $m / z(\%) 250\left(\mathrm{M}^{+}, 100\right), 223(20)$, 185 (30), 158 (15), 141 (20), $114(25), 82$ (10), 69 (15).

3.4. Synthesis of 4,7-diamino-3,6-di(thiophene-2-carbonyl)quinoline-8-carbonitrile (7).

A mixture of 2-aminoprop-1-ene-1,1,3-tricarbonitrile (1, $1.32 \mathrm{~g}, 0.01 \mathrm{~mol})$ and enaminone 2d $(0.01 \mathrm{~mol})$ in $\mathrm{AcOH}(10 \mathrm{~cm})$ and $0.2 \mathrm{gm}$ of $\mathrm{NH}_{4} \mathrm{OAc}$, was kept at reflux temperature for $3 \mathrm{hrs}$. The mixture was cooled and then poured onto ice-water. The solid so formed was collected by filtration and recrystallized from EtOH to give yellow crystals; Yield 88\%; m.p. 330-332 ${ }^{\circ} \mathrm{C}$; Anal. Calcd. for $\mathrm{C}_{20} \mathrm{H}_{12} \mathrm{~N}_{4} \mathrm{O}_{2} \mathrm{~S}_{2}$ (404): C, 59.40; H, 2.97; N, 13.86; O, 7.92; S, 15.84\%. Found: C, 59.50; H, 2.78; N, 13.96; O, 7.87; S, 15.89\%.; ${ }^{1} \mathrm{H}-\mathrm{NMR}: \delta$, ppm $=7.05$ (br. s, $\left.2 \mathrm{H}, \mathrm{NH}_{2}\right), 7.27(\mathrm{t}, J=4.0$, 1H, thienyl H-4'), 7.31 (t, $J=4.0,1 \mathrm{H}$, thienyl H-4"), 7.47 (s, 1H, H-5), 7.79 (d, 1H, J=3.2, thienyl H-3'), 7.85 (d, $3 \mathrm{H}, J=3.2$, thienyl H-3"\& $\mathrm{NH}_{2}$ ), 7.93 (d, $\left.1 \mathrm{H}, J=5.2, \mathrm{H}-5^{\prime}\right), 8.17$ (d, $\left.1 \mathrm{H}, J=5.2, \mathrm{H}-5 "\right), 8.98$ (s, $1 \mathrm{H}$, $\mathrm{H}-2) ;{ }^{13} \mathrm{C}-\mathrm{NMR}: \delta, \mathrm{ppm}=128.9,154.0(2 \mathrm{C}), 184.7(2 \mathrm{C}=\mathrm{O}), 77.1,98.3,103.9,115.85,118.0$, 129.2,131.6, 134.0, 135.7, 136.1, 140.6, 143.67, 156.1, 157.1, 160.4, 162.0; MS: $\mathrm{m} / z(\%) 404\left(\mathrm{M}^{+}\right.$, 100), 373 (10), $358(5), 319$ (20), 187 (5), 11 (20).

\subsection{General procedure for the synthesis of compounds 9a,b}

A mixture of 2-aminoprop-1-ene-1,1,3-tricarbonitrile (1, $1.32 \mathrm{~g}, 0.01 \mathrm{~mol})$ and enaminone 8a,b $(0.01 \mathrm{~mol})$ in $\mathrm{EtOH}(10 \mathrm{~mL})$ as a solvent was treated with piperidine (5 drops). The reaction mixture was refluxed for $4 \mathrm{hr}$. The mixture was cooled and then poured onto ice-water. The solid so formed was collected by filtration and recrystallized from $\mathrm{EtOH}$ to give yellow crystals.

6-Amino-2-dicyanomethylene-4-phenyl-2,3-dihydro-pyridine-3,5-dicarbonitrile (9a). Yield 82\%; m.p. 195-197 ${ }^{\circ} \mathrm{C}$; Anal. Calcd. for $\mathrm{C}_{16} \mathrm{H}_{8} \mathrm{~N}_{6}$ (284.28): C, 67.60; H, 2.84; N, 29.56\%. Found: C, 67.43; H, 2.61; N, 29.33\%; IR (KBr, cm $\left.{ }^{-1}\right): 3,467,3,323\left(\mathrm{NH}_{2}\right), 3,222(\mathrm{NH}), 2,314,2,219(4 \mathrm{CN}) ;{ }^{1} \mathrm{H}-\mathrm{NMR}: \delta$, ppm = 7.52-7.61 (7H, m, Ar-H, $\mathrm{NH}_{2}, \mathrm{D}_{2} \mathrm{O}$ exchangeable), $8.19\left(1 \mathrm{H}\right.$, br. s, NH, $\mathrm{D}_{2} \mathrm{O}$ exchangeable); ${ }^{13} \mathrm{C}-\mathrm{NMR}: \delta, \mathrm{ppm}=160.35,158.81,158.71,133.82,130.47,128.76(2 \mathrm{C}), 128.67,128.36(2 \mathrm{C})$, 116.10, 114.60, 113.5, 95.29, 89.17; MS: m/z (\%) $284\left(\mathrm{M}^{+}, 100\right), 257$ (25), 219 (10), 165 (25), 127 (10), 77 (5).

2-[6-Amino-3-aminoethynyl-5-cyano-4-(4-methoxy-phenyl)-1H-pyridin-2-ylidene]malononitrile (9b). Yield 82\%; m.p. 248-250 ${ }^{\circ} \mathrm{C}$; Anal. Calcd. for $\mathrm{C}_{17} \mathrm{H}_{10} \mathrm{~N}_{6} \mathrm{O}$ (314.31): C, 64.96; H, 3.21; N, 26.74\%. Found: C, 65.01; H, 3.22; N, 26.44\%; IR (KBr, cm $\left.{ }^{-1}\right): 3,423,3,327\left(\mathrm{NH}_{2}\right), 3,212(\mathrm{NH}), 2,187,2,150$ $(3 \mathrm{CN}) ;{ }^{1} \mathrm{H}-\mathrm{NMR}: \delta, \mathrm{ppm}=3.82\left(\mathrm{~s}, 3 \mathrm{H}, \mathrm{OCH}_{3}\right), 6.86\left(\mathrm{br}, 2 \mathrm{H}, \mathrm{NH}_{2}, \mathrm{D}_{2} \mathrm{O}\right.$ exchangeable), $7.04(2 \mathrm{H}, \mathrm{d}$, $\left.J=8.0, \mathrm{H}-3^{\prime}, 5^{\prime}\right), 7.35$ (2H, d, $\left.J=8.0, \mathrm{H}-2^{\prime}, 6^{\prime}\right), 8.22$ (1H, br. s, NH, $\mathrm{D}_{2} \mathrm{O}$ exchangeable); ${ }^{13} \mathrm{C}-\mathrm{NMR}: \delta$, $\mathrm{ppm}=162.95,160.23,159.47,158.97,133.45,130.19(2 \mathrm{C}), 127.80,121.39,117.24,116.92,113.82$ (2C), 85.26, 80.48, 55.31; MS: m/z (\%) $284\left(\mathrm{M}^{+}, 100\right), 257$ (25), 219 (10), 165 (25), 127 (10), 77 (5). 


\subsection{Synthesis of 3,5-Diaminobiphenyl-2,4,6-tricarbonitrile (10)}

A mixture of 2-aminoprop-1-ene-1,1,3-tricarbonitrile (1, $1.32 \mathrm{~g}, 0.01 \mathrm{~mol})$ and benzylidenemalononitrile $(\mathbf{8}, 0.01 \mathrm{~mol})$ in $\mathrm{AcOH}(10 \mathrm{~cm})$ and $0.2 \mathrm{gm}$ of $\mathrm{NH}_{4} \mathrm{OAc}$, was kept under reflux temperature for $3 \mathrm{hr}$. The mixture was cooled and then poured onto ice-water. The solid so formed was collected by filtration and recrystallized from $\mathrm{EtOH}$ to give yellow crystals; yield $80 \%$; m.p. $290-292{ }^{\circ} \mathrm{C}$; Anal. Calcd. for $\mathrm{C}_{15} \mathrm{H}_{9} \mathrm{~N}_{5}$ (259.27): C, 69.49; H, 3.50; N, 27.01\%. Found: $\mathrm{C}, 69.62 ; \mathrm{H}, 3.34$; $\mathrm{N}$, 27.17\%; IR (KBr, cm $\left.{ }^{-1}\right): 3,371,3,305\left(\mathrm{NH}_{2}\right), 3,265,3,213\left(\mathrm{NH}_{2}\right), 2,218(3 \mathrm{CN}) ;{ }^{1} \mathrm{H}$ NMR: $\delta$, ppm $=4.51$ (br. s, $4 \mathrm{H}, 2 \mathrm{NH}_{2}, \mathrm{D}_{2} \mathrm{O}$ exchangeable), 7.41-7.44 (2H, m, H-3',5'), 7.50-7.53 (3H, m, H-2',4',6'); ${ }^{13} \mathrm{C}$ NMR: $\delta, p p m=160.92$ (2C), 157.07, 135.08, 129.92, 128.49 (2C), $128.21(2 \mathrm{C}), 115.61$ (2C), 85.65, 81.06, 75.95, 66.31; MS: m/z (\%) 259 (M+100), 234 (20), 205 (15), 165 (20), 127 (10), 77 (50).

\subsection{Synthesis of 2-(4-Amino-7-cyano-3,9-diphenylpyrido[1,2-a]pyrimidin-6-ylidene)-malononitrile (14)}

A mixture of 2-aminoprop-1-ene-1,1,3-tricarbonitrile (1, $1.32 \mathrm{~g}, 0.01 \mathrm{~mol})$ and 2-phenyl-3piperidin-1-yl-acrylonitrile $(\mathbf{1 1}, 0.01 \mathrm{~mol})$ in dioxane $(10 \mathrm{~mL})$ was kept under reflux temperature for 34 hrs. The mixture was cooled and then poured onto ice-water. The solid so formed was collected by filtration and recrystallized from $\mathrm{AcOH}$ to give yellow crystals; yield 85\%; m.p. $270-272{ }^{\circ} \mathrm{C}$; Anal. Calcd. for $\mathrm{C}_{24} \mathrm{H}_{14} \mathrm{~N}_{6}$ (386.42): C, 64.60; H, 3.65; N, 21.75\%. Found: C, 64.48; H, 3.55; N, 21.90\%; IR $\left(\mathrm{KBr}, \mathrm{cm}^{-1}\right): 3,383,3,186\left(\mathrm{NH}_{2}\right), 2,237,2,196(3 \mathrm{CN}) ;{ }^{1} \mathrm{H}-\mathrm{NMR}: \delta, \mathrm{ppm}=6.82\left(2 \mathrm{H}, \mathrm{br} . \mathrm{s}, \mathrm{NH}_{2}, \mathrm{D}_{2} \mathrm{O}\right.$ exchangeable), 7.24-7.52 (m, 12H, Ar-H); MS: $m / z$ (\%) $386\left(\mathrm{M}^{+}, 10\right), 379$ (40), 337 (70), 319 (100), 278 (95), 259 (35), 251 (30), 210 (20), 179 (20), 155 (10), 140 (25), 115 (15), 140 (25), 115 (10), 77 (15), 59 (20).

\subsection{Synthesis of 2-amino-4-(dimethylamino)buta-1,3-diene-1,1,3-tricarbonitrile (16)}

A mixture of 2-aminoprop-1-ene-1,1,3-tricarbonitrile (1, $1.32 \mathrm{~g}, 0.01 \mathrm{~mol})$ and DMFDMA (1.19 g, $0.01 \mathrm{~mol})$ in dioxane $(10 \mathrm{~mL})$ was stirred for $3-4 \mathrm{hrs}$. The mixture then poured onto ice-water. The solid, so formed, was collected by filtration and recrystallized from EtOH to give yellow crystals; yield 90 \% m.p. $189-190{ }^{\circ} \mathrm{C}$. Anal. Calcd. for $\mathrm{C}_{9} \mathrm{H}_{9} \mathrm{~N}_{5}$ (187.2): C, 57.74; H, 4.85; N, 37.41\%. Found: C, 57.61; H, 4.57; N, 37.19\%; IR $\left(\mathrm{KBr}, \mathrm{cm}^{-1}\right): 3,344,3,221\left(\mathrm{NH}_{2}\right), 2,208,2,193(3 \mathrm{CN})$; ${ }^{1} \mathrm{H}$ NMR $\left(400 \mathrm{MHz}, \mathrm{DMSO}-\mathrm{d}_{6}\right): \delta, \mathrm{ppm}=2.50\left(3 \mathrm{H}, \mathrm{s}, \mathrm{CH}_{3}\right), 2.57\left(3 \mathrm{H}, \mathrm{s}, \mathrm{CH}_{3}\right), 7.19$ (br. s, $2 \mathrm{H}, \mathrm{NH}_{2}, \mathrm{D}_{2} \mathrm{O}$ exchangeable), $8.54(1 \mathrm{H}, \mathrm{s}$, olefinic $\mathrm{CH})$; MS: $m / z(\%) 187\left(\mathrm{M}^{+}, 100\right), 172$ (30), 159 (35), 144 (25), 122 (60), 117 (20), 97 (15), 95 (15), 81 (20), 67 (20), 57 (30).

\subsection{Synthesis of 4,6-diamino-2H-pyrazolo[3,4-b]pyridine-5-carboxamide hydrazone (17)}

A mixture of $13(1.87 \mathrm{~g}, 0.01 \mathrm{~mol})$ and hydrazine monohydrate $(1.00 \mathrm{~g}, 0.02 \mathrm{~mol})$ in EtOH $(20 \mathrm{~mL})$ was refluxed for 3-4. The mixture then poured onto ice-water. The solid so formed was collected by filtration and recrystallized from $\mathrm{EtOH}$ to give a faint yellow product; yield $87 \%$; m.p. $210-212{ }^{\circ} \mathrm{C}$; Anal. Calcd. for $\mathrm{C}_{7} \mathrm{H}_{10} \mathrm{~N}_{8}$ (206.21): C, 40.77; H, 4.89; N, 54.34\%. Found: C, 40.58; H, 4.65; N, $54.05 \%$; IR $\left(\mathrm{KBr}, \mathrm{cm}^{-1}\right)$ : complicated signals from 3,402 to 3,156 for $(\mathrm{NH})$ and $\left(4 \mathrm{NH}_{2}\right) ;{ }^{1} \mathrm{H}-\mathrm{NMR}$ : $\delta$, ppm $=5.34$ (2H, br. s, $\mathrm{NH}_{2}, \mathrm{D}_{2} \mathrm{O}$ exchangeable), $5.97\left(2 \mathrm{H}\right.$, br. s, $\mathrm{NH}_{2}, \mathrm{D}_{2} \mathrm{O}$ exchangeable), $7.10(2 \mathrm{H}$, br. s, $\mathrm{NH}_{2}, \mathrm{D}_{2} \mathrm{O}$ exchangeable), $7.29\left(2 \mathrm{H}\right.$, br. s, $\mathrm{NH}_{2}, \mathrm{D}_{2} \mathrm{O}$ exchangeable), $8.00(1 \mathrm{H}, \mathrm{s}, \mathrm{H}-3), 8.82(1 \mathrm{H}$, 
br. s, NH, $\mathrm{D}_{2} \mathrm{O}$ exchangeable); MS: m/z (\%) $106\left(\mathrm{M}^{+}, 100\right), 190$ (95), 174 (100), 159 (75), 145 (40), 109 (35), 92 (40), 77 (60), 67 (100).

\section{Conclusions}

We could successfully utilize $\mathbf{1}$ as precursor to a variety of polyfunctionally substituted aminoaromatics that seem of value as potential precursors to dyes and pharmaceuticals.

\section{References}

1. Fahmy, S.M.; Abed, N.M.; Mohareb, R.M.; Elnagdi, M.H. Activated nitriles in heterocyclic synthesis: Novel synthesis of pyridazines, pyridines, and isoxazoles. Synthesis 1982, 6, 490-493.

2. Elmoghayar, M.R.H.; Ibraheim, M.K.A.; Elghandour, A.H.H.; Elnagdi, M.H. A novel synthesis of thiazolo[3,2-a] pyridine derivatives. Synthesis 1981, 8, 635-637.

3. Elnagdi, M.H. Reaction with $\alpha$-cyanoethylhydrazine. I. Route for the preparation of pyrazolo[1,5a]pyrimidines and pyrrolo[1,2-b]pyrazoles. Tetrahedron 1974, 30, 2791-2796.

4. Erian, A.W. The chemistry of $\beta$-enaminonitriles as versatile reagents in heterocyclic synthesis. Chem. Rev. 1993, 93, 1991-2005.

5. Al-Matar, H.M.; Khalil, K.D.; Meier, H.; Kolshorn, H.; Elnagdi, M.H. Chitosan as heterogeneous catalyst in Michael additions: The reaction of cinnamonitriles with active methylene moieties and phenols. ARKIVOC 2008, 16, 288-301.

6. Al-Awadi, N.A.; Abdelkhalik, M.M.; Abdelhamid, I.A.; Elnagdi, M.H. Pyrolytic methods in organic synthesis: Novel routes for the synthesis of 3-oxoalkanenitriles, 2-acyl anilines, and 2aroyl anilines. Synlett 2007, 19, 2979-2982.

7. Saleh, M.A.; Moustafa, S.M.; Elnagdi, M.H. Green Synthetic approaches: Solventless synthesis of polyfunctionally substituted aromatics as potential versitile building blocks in organic synthesis utilizing enaminones and enaminonitriles as precursors. Green Chem. Lett. Rev. 2010, in print.

8. Taylor E.C., McKillop, A. The Chemistry of Cyclic Enaminonitriles and o-Amino-nitriles; Interscience; New York, NY, USA, 1970; p. 415.

9. Freeman, F. Chemistry of malononitrile. Chem. Rev. 1969, 69, 591.

10. Fatiadi, A.J. New applications of malononitrile in organic chemistry-part I. Synthesis 1978, 165.

11. Hassanien, A.A.; Abu Zeid, A.; Ghozlan, S.A.; Elnagdi, M.H. Enaminones as Building Blocks in Organic Synthesis: A Novel Route to Polyfunctionally Substituted Benzonitriles, Pyridines, Enylbenzotriazoles and Diazepines. J. Heterocycl. Chem. 2003, 40, 225-228.

12. Helmy, N.M. Synthesis of Polyfunctional Heterocycles Using Microwave As Environmentally Friendly Technique. PhD Thesis, Cairo University, Cairo, Egypt, 2006.

Sample Availability: Samples of the compounds 3a, 4a-d, 7, 9a-b, 10, 14 and 17 are available from the authors.

(C) 2011 by the authors; licensee MDPI, Basel, Switzerland. This article is an open access article distributed under the terms and conditions of the Creative Commons Attribution license (http://creativecommons.org/licenses/by/3.0/). 